\title{
USO DE HERRAMIENTAS PARA LA GESTIÓN DEL CONOCIMIENTO EN LA ETAPA SECUNDARIA OBLIGATORIA
}

Use of tools for Knowledge Management in Compulsory Secondary Education Uso de ferramentas de gestão do conhecimento no Ensino Médio

Durante la realización de este estudio, S. García-Martín recibió, en concurrencia competitiva, una ayuda para la realización de estudios de doctorado de la Universidad de León (convocatoria 2015).

\section{Sheila García-Martín (1)}

Isabel Cantón Mayo (2)

(1) Universidad de León, España. Teléfono: +34 987295175. Correo electrónico: sgarcm@unileon.es

(2) Universidad de León, España. Teléfono: +34 987291029. Correo electrónico: icanm@unileon.es

\begin{abstract}
Resumen
La gestión del conocimiento se entiende como el conjunto de procesos orientados al desarrollo organizacional y/o personal y cuyos factores principales son las personas, los procesos y la tecnología, donde se incluyen las herramientas para la gestión del conocimiento. Para conocer la opinión de los alumnos de Educación Secundaria Obligatoria respecto al uso de herramientas para la gestión del conocimiento en cuatro asignaturas de la etapa, se diseñó y validó un cuestionario que fue aplicado a 397 estudiantes. Los resultados muestran que la mayoría está conforme con el uso que se hace de las herramientas y destacan sus posibilidades; como la cantidad de información a la que pueden acceder, al mismo tiempo; subrayan las dificultades para un correcto uso. La formación previa y continua se configura, entonces, como un elemento indispensable.

Palabras clave: Gestión del conocimiento; herramientas; educación secundaria obligatoria
\end{abstract}




\begin{abstract}
Knowledge Management is understood as the set of processes oriented to organizational and/or personal development and whose main factors are people, processes and technology, where tools for Knowledge Management are included. In order to know the opinion of the students of Compulsory Secondary Education regarding the use of tools for Knowledge Management in four subjects of the stage, a questionnaire was designed and validated and it was applied to 397 students. The results show that the majority is satisfied with the use of tools and they emphasize their possibilities as the quantify of information they can access, at the same time, they underline the difficulties for a correct use. Previous and continuous formation is configured as an essential element.
\end{abstract}

Keywords: Knowledge management; tools; compulsory secondary education

\title{
Resumo
}

A gestão do conhecimento é entendido como o conjunto de processos para o desenvolvimento organizacional e/ou pessoal e cujos fatores principais são as pessoas, procesos e tecnologia, onde as ferramentas de gestão do conhecimento estão incluídas. Para saber a opinião dos estudantes de Ensino Médio em relação ao uso de ferramentas para gestão do conhecimento em quatro matérias da etapa, foi desenvolvido, validado e aplicado um questionário a 397 alunos. Os resultados mostram que a maioria está satisfeita com a utilização das ferramentas e destacam seu potencial como a quantidade de informação que pode ser acessado, ao mesmo tempo, eles sublinham as dificuldades para o uso adequado. Formação prévia e contínua é configurada como un elemento indispensável.

Palavras-chave: Gestão do conhecimento; ferramentas; ensino médio

\section{Introducción}

La gestión del conocimiento se entiende como un conjunto de procesos sistemáticos orientados al desarrollo organizacional y/o personal que generan una ventaja competitiva para la organización y/o el individuo (Rodríguez, 2006).

Los factores esenciales de la Gestión del Conocimiento son las personas, los procesos y la tecnología, es en ésta última donde se ubican las herramientas para la 
gestión del conocimiento. Las diferentes revisiones llevadas a cabo para estudiar los efectos de la implementación de procesos de gestión del conocimiento y de herramientas en las organizaciones educativas, muestran que su desarrollo resulta efectivo y positivo, no sólo en el rendimiento organizativo de la escuela, sino también, en el rendimiento escolar de los estudiantes (García-Martín \& Cantón-Mayo, 2016; García-Martín, Hidalgo \& Cantón, 2016, Palazón, 2013), en su satisfacción y en el aumento de su participación en el proceso de enseñanza-aprendizaje (Espigares \& García, 2010).

\section{Método}

Desarrollamos un estudio descriptivo-interpretativo en el que se diseñó, validó y aplicó un cuestionario ad hoc a 397 alumnos de ESO de dos centros públicos de la ciudad de León, previa autorización de la Junta de Castilla y León y autorización paterna. Con el instrumento se trató de conocer, entre otras variables, la opinión de los alumnos de secundaria respecto al uso de herramientas para la gestión del conocimiento en cuatro asignaturas de la etapa.

\section{Resultados y discusión}

La muestra estuvo conformada por 190 chicos y 207 chicas matriculados en uno de los cuatro cursos de ESO de dos centros públicos de la ciudad de León, distribuidos porcentualmente como muestran las figuras 1 y 2 .

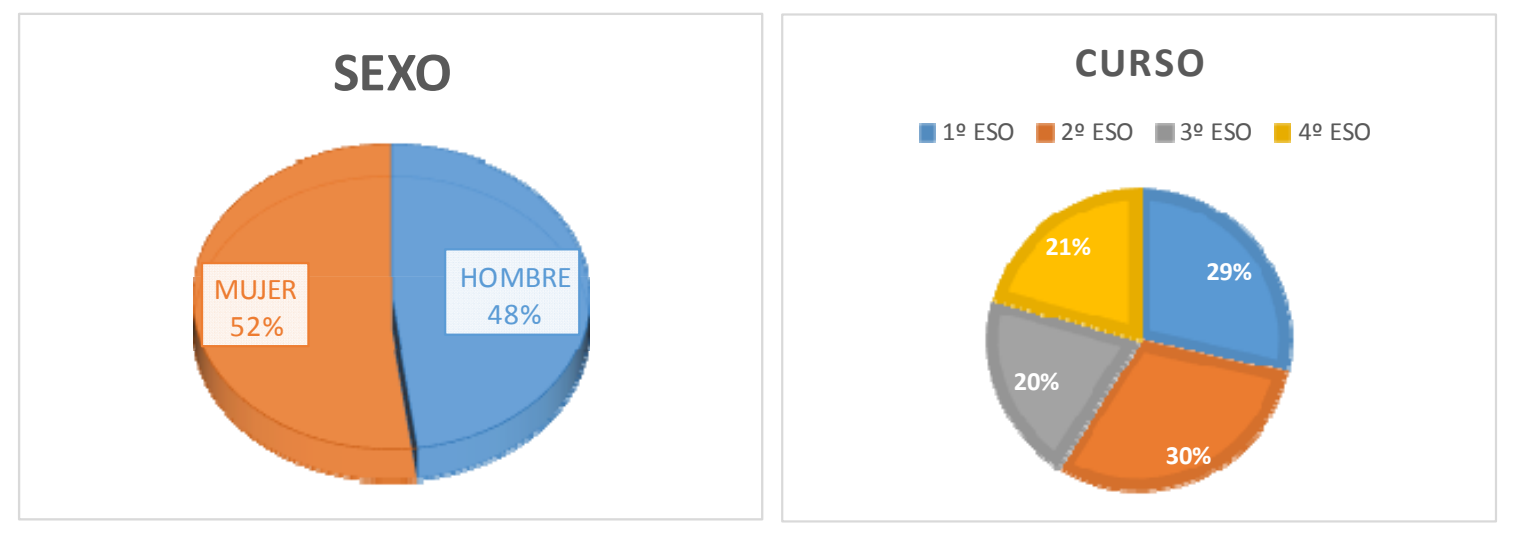

Figura 1.

Distribución por sexo
Figura 2.

Distribución por curso 
Los estudiantes expusieron su opinión sobre el uso que se hace de cinco herramientas para la gestión del conocimiento: Motores de búsqueda, wikis, podcast, blog y mensajería instantánea en las clases de Ciencias Sociales, Matemáticas, Lengua y Literatura e Inglés. Se les preguntó si en las clases de esas cuatro asignaturas se utilizaban dichas herramientas y si ellos estaban de acuerdo con el uso que se hacía de las mismas o consideraban que debía hacerse un mayor o menor uso y el porqué de su respuesta.

Los resultados obtenidos muestran que se usan estas herramientas, especialmente los motores de búsqueda (58\%), en las asignaturas. Los estudiantes, en su mayoría, están conformes con el uso que se hace de estas herramientas. Pues como se puede observar en el siguiente gráfico, la mayor parte de los alumnos aboga por seguir utilizando las herramientas en la misma medida que hasta ahora.

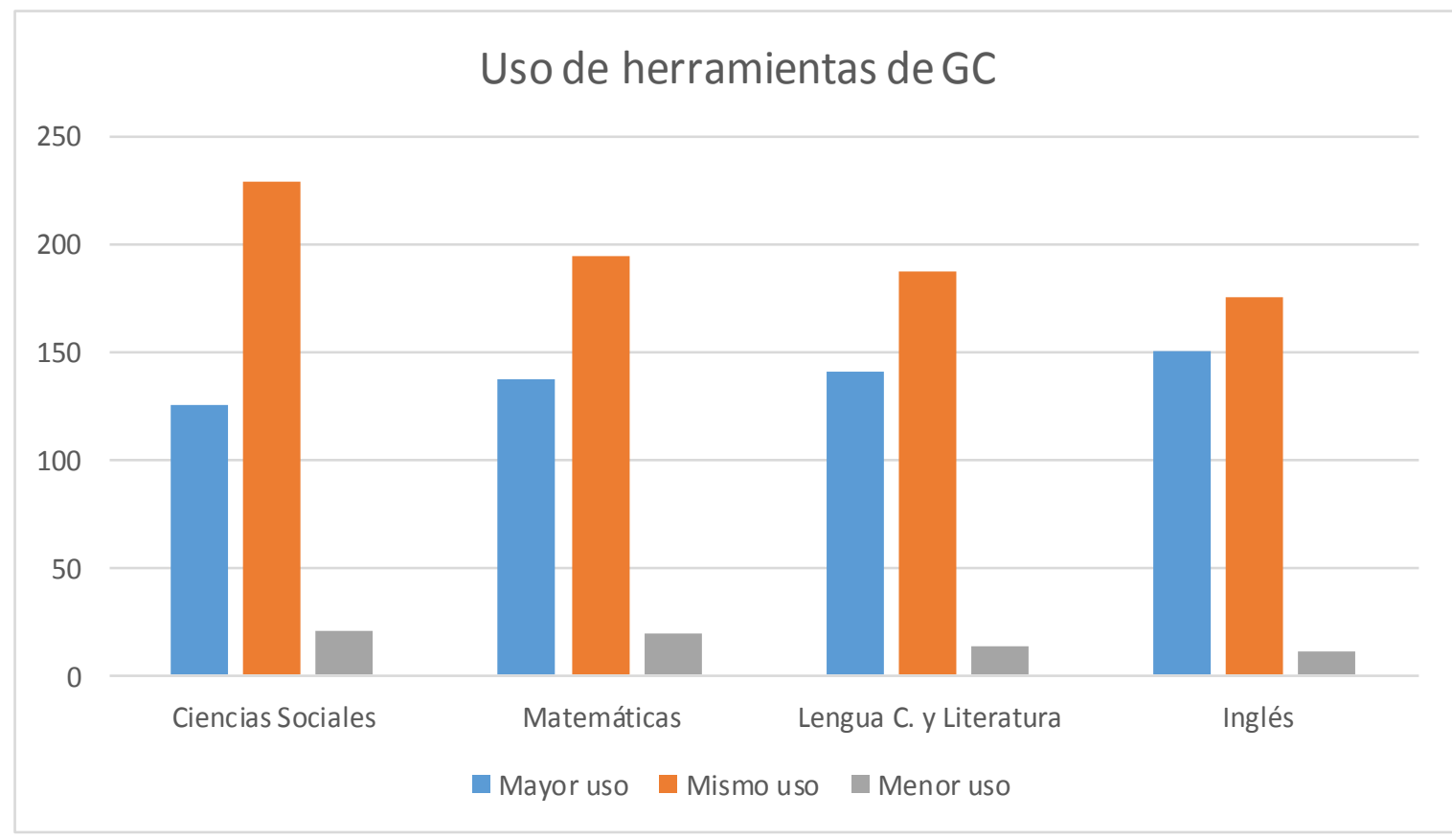

Figura 3.

Opinión de los alumnos respecto al uso de herramientas de GC en cuatro asignaturas

Entre las razones que los alumnos expresan para justificar el mismo uso de herramientas destacan que la clase está bien como está; p.33: “nos va bien”, p.76: “el funcionamiento actual es bueno”, p.200: “porque van bien las clases”. Entre los estudiantes que expresan su convicción por un mayor o menor uso de herramientas, se recogen sus razones en las tablas 1 y 2. 
Tabla 1.

Razones para un mayor uso de herramientas de Gestión del Conocimiento

\begin{tabular}{lc}
\hline & $\mathbf{\%}$ \\
\hline 1. Mayor aprendizaje. Más información. & $45,6 \%$ \\
2. Diversión. Clases más amenas & $36,9 \%$ \\
3. Mejora de las notas. Mayor rendimiento. & $10,8 \%$ \\
4. Mayor aprovechamiento de las TIC & $6,5 \%$
\end{tabular}

Porque facilita el aprendizaje y porque hay más información: p.3: “así aprendemos más cosas”, p.20: “mayor información, recursos audiovisuales en interacción”; p. 34:” para aclarar mejor lo conceptos explicados”, p. 65: “porque así encontramos más información en distintas fuentes” es la razón principal por la que los alumnos defienden un mayor uso de herramientas. Seguida de la diversión que supone utilizar dichas herramientas en la clase: p. 68: “porque así las clases serían más divertidas y más amenas”, p.90: “porque es más divertido”, p.114: “porque las clases serían más entretenidas”.

Tabla 2.

Razones para un menor uso de herramientas de Gestión del Conocimiento

\begin{tabular}{lc}
\hline & $\%$ \\
\hline 1. Confusión y falta de fiabilidad información. & $43,5 \%$ \\
2. Uso excesivo de las herramientas & $31,2 \%$ \\
3. Distracción. & $14,7 \%$ \\
4. Otras. & $10,6 \%$ \\
\hline
\end{tabular}

La confusión, la falta de fiabilidad de la información o la inexperiencia para encontrar información de calidad es la razón principal con la que los alumnos justifican un uso menor de las herramientas P.1: “a lo mejor la información es falsa”, p.105: "yo me lío con los videos prefiero que me lo explique la profesora", p.312: "porque no hay nada de utilidad que exista a día de hoy”. Asimismo, destacan que ya hacen un uso suficiente de las herramientas fuera del centro por lo que dentro del mismo no sería necesario; p 78: “porque si estuviésemos todo el rato con internet no tendríamos vida”, p.95: "porque si no tenemos mucha adicción” además destacan la distracción que suponen; p.346: "porque te aparta del estudio y te distraes pensando en ello".

\section{Conclusiones}

Como observamos en los resultados, en la etapa Secundaria Obligatoria se hace uso de herramientas para la Gestión del Conocimiento y para la mayoría de los estudiantes encuestados, el uso que se hace es suficiente pero también, necesario. Es preciso 
destacar que los alumnos que abogan por un mayor o menor uso lo hacen con fuertes razones que debemos valorar como docentes e investigadores. Por un lado, como recogen los estudiantes, estas herramientas les proveen de mucha información, son divertidas y útiles para ellos, por otro lado, un correcto uso lleva consigo formación previa y continua para poder localizar información de calidad y para conocer las aplicaciones didácticas de estas herramientas en todas las asignaturas de la etapa.

\section{Referencias}

Espigares, M.J., \& García, R. (2010). Evaluación de un modelo de gestión del conocimiento educativo-musical: El modelo Bordón. Leeme. Lista Europea Electrónica de Música en la Educación, 25, 49-64.

García-Martín, S., \& Cantón-Mayo, I. (2016). La adecuada gestión del conocimiento en las escuelas. En J.L. Castejón (Comp.). Psicología y Educación. Presente y futuro (pp. 2357-2363). Madrid: ACIPE-Asociación Científica de Psicología y Educación.

García-Martín, S., Hidalgo, S., \& Cantón, I. (2016). La gestión del conocimiento en instituciones educativas. Implicaciones en el éxito escolar. En J. Gairín (Ed.). Aprendizaje situado y aprendizaje conectado: implicaciones para el trabajo. Barcelona: Wolters Kluwer.

Rodríguez, D. (2006). Modelos para la creación y gestión del conocimiento: una aproximación teórica. Educar, 37, 25-39. 